\title{
Anti-Cancer Drug Validation: the Contribution of Tissue Engineered Models
}

\author{
Mariana R. Carvalho ${ }^{1,2}$ • Daniela Lima ${ }^{1,2}$ - Rui L. Reis ${ }^{1,2}$. \\ Joaquim M. Oliveira ${ }^{1,2} \cdot$ Vitor M. Correlo ${ }^{1,2}$
}

(C) Springer Science+Business Media New York 2017

\begin{abstract}
Drug toxicity frequently goes concealed until clinical trials stage, which is the most challenging, dangerous and expensive stage of drug development. Both the cultures of cancer cells in traditional 2D assays and animal studies have limitations that cannot ever be unraveled by improvements in drug-testing protocols. A new generation of bioengineered tumors is now emerging in response to these limitations, with potential to transform drug screening by providing predictive models of tumors within their tissue context, for studies of drug safety and efficacy. Considering the NCI60, a panel of 60 cancer cell lines representative of 9 different cancer types: leukemia, lung, colorectal, central nervous system (CNS), melanoma, ovarian, renal, prostate and breast, we propose to review current "state of art" on the 9 cancer types specifically addressing the $3 \mathrm{D}$ tissue models that have been developed and used in drug discovery processes as an alternative to complement their study.
\end{abstract}

Keywords Cancer $\cdot$ Tissue engineering $\cdot$ Biomaterials $\cdot 3 \mathrm{D}$. Drug discovery

Joaquim M. Oliveira and Vitor M. Correlo are Senior authors

Rui L. Reis

rgreis@dep.uminho.pt

1 3B's Research Group - Biomaterials, Biodegradables and Biomimetics, Headquarters of the European Institute of Excellence on Tissue Engineering and Regenerative Medicine, University of Minho, AvePark, 4806-909 Taipas, Guimarães, Portugal

2 ICVS/3B's - PT Government Associate Laboratory, Braga/ Guimarães, Portugal

\section{Introduction}

Cancer is a major cause of morbidity and mortality worldwide, with disease burden estimated to increase in the coming decades. Nonetheless, we are living through an exciting era for cancer drug discovery and development, full of enormous opportunities and challenges. Scientific advances in drug discovering technologies such as genomic methods, drug screening technologies, tissue engineering and microfluidics lead to the witnessing of a host of technology changes and new insights into the biology of neoplasia. Despite these advances, testing of new molecules in the clinical stages of development remains a lengthy process associated with a high failure rate [1].

Cancer is a unique term that designates a complex and diversified disease that calls for at least 100 different types of cancer and can virtually affect any part of the body [2]. Malignant tumors or neoplasms may also be used to refer to this condition, whose main feature is the rapid proliferation of abnormal cells, which can then invade other tissues. This process is known as metastasis and it is one of the main causes of cancer deaths [2].

Cancer therapy is a rather young medical discipline which, from 1950's up to now has resulted in the approval of nearly 183 anti-cancer drugs $[3,4]$. It also poses an unprecedented challenge to big pharmaceutical industry since the costs for the development of a single anti-cancer drug can reach up to US\$ 1 billion $[5,6]$. The process of drug development involves critical and systematic steps with many drawbacks and turnarounds. It is estimated that per 10,000 compounds analyzed for different applications, only one reaches approval [6]. In the case of anticancer drugs, the attrition rates are even higher, since only $5 \%$ of substances passing clinical testing are later licensed and many others commercially available are later withdrawn from the market [7]. This high attrition rates 
can be mainly attributed to the inexistence of adequate preclinical models to test their efficacy and toxicity [7]. It is therefore urgent to develop new nanopharmaceuticals release systems as well as efficient drug screening methodologies that are able to mimic the behavior in the human body and predict the future success of specific drugs.

Since 1995, the process of testing a new potential anticancer drug involves an initial pre-screening using 3 cell lines, MCF-7 breast cancer, NCI-H460 large-cell lung cancer and SF268 glioblastoma cancer cell lines [5, 8]. Only then, if the substance inhibits growth of at least one of the cell lines, it proceeds to be tested using the NCI60, a panel of 60 cancer cell lines representative of 9 different cancer types: leukaemia, lung, colorectal, central nervous system (CNS), melanoma, ovarian, renal, prostate and breast $[8,9]$. Up to now, the NCI60 panel has allowed testing of more than 70,000 compounds, including all chemotherapeutic agents currently used [10]. Preoccupying evidence is arising on whether commonly used cancer cell lines are representative of the tumors they intend to mimic [11].

The most frequently used model for in vitro study of mammalian cells is the monolayer of cells. Even though the twodimensional (2D) model has indubitable given us a significant knowledge amount in biological research, it has certain limitations that have encouraged the development of threedimensional (3D) models. The complex nature of tumors and the influence of the surrounding microenvironment which 2D cell cultures alone, cannot recapitulate. The lack of such complexity may provide inaccurate information regarding the efficacy of such treatments, thus jeopardizing the development of appropriate chemotherapy drugs (Fig. 1). Additionally, there are basic differences between $2 \mathrm{D}$ and $3 \mathrm{D}$ cultures that emphasize the need to use both screening systems. The morphology of cells is affected by its growth medium: in 2D, cancer cells display an unnatural morphology, while in 3D they adopt a rounded shape, forming clusters that are suggestive of tumors in vivo $[13,14]$. Growth rates also differ according to their environment, as cancer cells grow faster in $2 \mathrm{D}$ than in $3 \mathrm{D}$ [15]. According to some mathematical models, the latter reflects better the in vivo growth rates of cancer cells [16]. Molecular differences (such as differential gene expression profiles in key genes involved in angiogenesis, cell migration and invasion) have also been highlighted between cancer cells grown in 2D or in 3D [17-19]. Finally, numerous reports have emphasized important differences in drug responses of cancer cells grown in 2D compared to 3D [20-22]. Although these results are highly dependent on the type of cell and drug, an overall higher resistance to treatments has been observed in 3D tests, which may provide a possible explanation to drug resistance phenomena observed in some patients. It is the added dimensionality of $3 \mathrm{D}$ cultures that is the critical feature leading to the differences in cellular responses because not only it influences the spatial organization of the cell surface receptors engaged in interactions with surrounding cells, but it also induces physical constraints to cells. Nutrients, growth factors or drugs may not be able to fully penetrate the 3D system to reach cells near the core, more resistant to treatment compared to those in 2D culture system, often being better predictors of in vivo drug responses. Also, as cytostatic drugs mainly target proliferating cells, cancer cell dormancy is considered as a major resistance mechanism to this class of anti-cancer drugs, only reproduced by 3D models dormant tumor regions [23].

In this paper we review current "state of art" on the 9 cancer types represented by the NCI60 cell line panel, specifically addressing the $3 \mathrm{D}$ tissue engineering models have been developed and used in drug discovery processes as an alternative to complement their study. The potential of using $3 \mathrm{D}$ cell models for anti-cancer drug screening is here critically assessed, and its main advantages and limitations dissected. Furthermore, we also compile current information regarding anti-cancer drug testing in $3 \mathrm{D}$ versus $2 \mathrm{D}$ and critically assess their potential impact in the discovery processes of novel chemotherapy drugs.

\section{Tissue Engineering}

Tissue engineering aims at providing biological substitutes to restore or replace tissue and organ function, as an alternative to currently available conventional options, such as transplantation, surgical reconstruction, artificial prosthesis or supplementation of the metabolic products [24].

3D culture systems can be subdivided as scaffold-free or scaffold-based (of natural or synthetic origin) methods [25, $26]$. One of the most commonly used scaffold-free 3D cancer models are multicellular tumor spheroids, which are formed due to the ability of cells in suspension to self-assemble in the absence of an attachment surface or scaffold [27]. These are simple and easy to produce and can be constituted of one or more cell types. Their small size and the absence of vascularization create limitations to nutrients and oxygen diffusion, requiring cells of its core to adopt a glycolytic metabolic activity which causes an increasing $\mathrm{pH}$ gradient towards the outside (Fig. 2) [28]. This mimics what happens with tumors in vivo, which display regions of hypoxia and of acidity according to the distance to tumor blood vessels, as can be seen in Fig. 2 [28-30]. Spheroids can be fabricated using several methods: hanging drop method, in which cells can aggregate freely at the bottom of an inverted cell suspension drop; spinner flasks method, which provides constant agitation, allowing spontaneous cell aggregation; static liquid overlay technique, which enables spheroid formation by preventing cell adhesion to the growth substrate; centrifugation; and growing cells on non-adherent micropatterned surfaces in microfabricated devices to favor their aggregation [31]. 
Fig. 1 Schematic representation of the potential role of 3D TE models in cancer research, namely in drug development as a complement to $2 \mathrm{D}$ cell culture and animal models. The correlation here represented refers to the Pearson Correlation Coefficient comparing gene expression in spontaneous SCC tumors with 3D organotypic models, mouse and 2D models. Adapted from Ridky et al. [12]

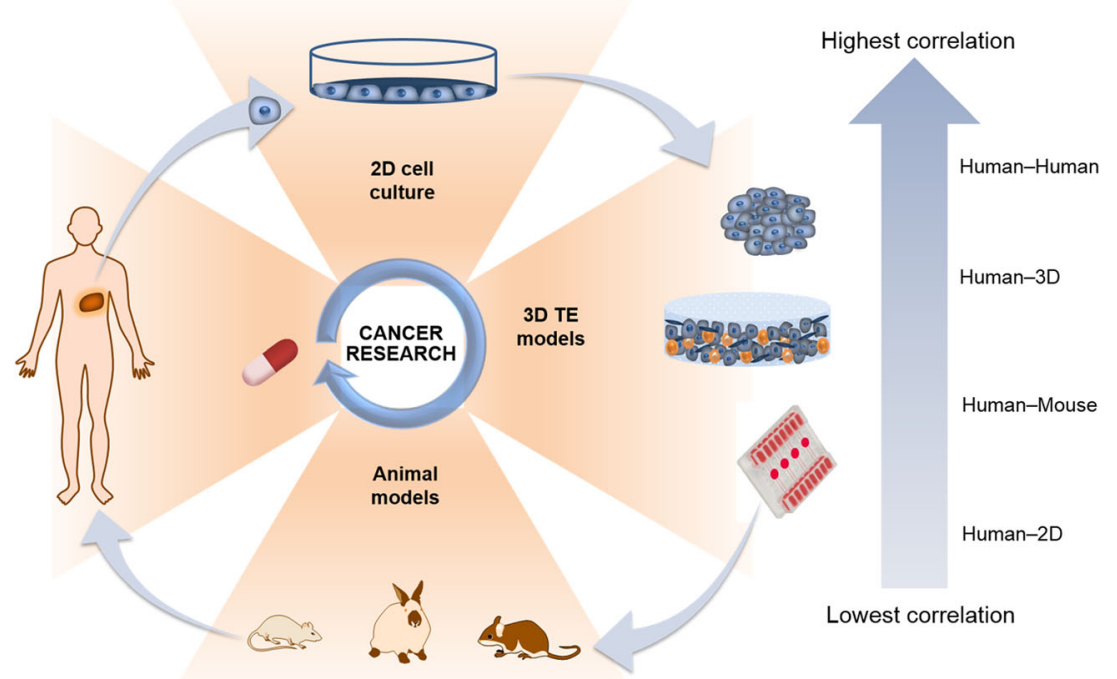

Other studies use scaffold-based methods for building 3D tumor models, either of synthetic or natural origin [26, 28]. There are still manifest limitations associated to any of these strategies to build 3D cancer models, which need to be optimized to better mimic the complex tumor characteristics and its interactions with the surrounding stroma, and ultimately be incorporated in routine tests for testing anti-cancer drugs. Another promising technology arising in drug discovery for cancer research is microfluidics. Microfluidics allow the fabrication of $3 \mathrm{D}$ architectures with controlled spatial relations between cells inside a chip, the presence of flow-induced signaling and transduction, and the capacity to introduce the chemical gradients necessary to reproduce the architecture of the in vivo microenvironment [32]. This technology, generally combined with Tissue Engineering, has provided instrumental data and previously unmeasurable or unobservable informations about cancer-related processes, such as angiogenesis and metastasis, and have proved instrumental in drug discovery [26].

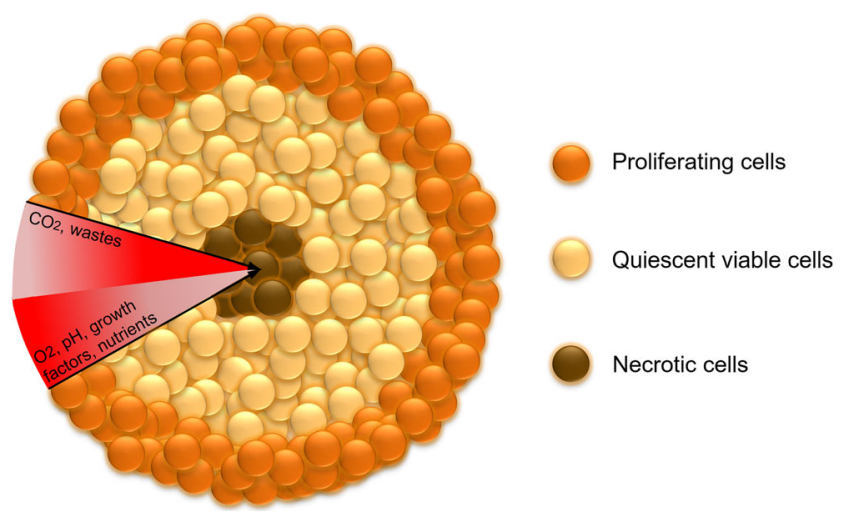

Fig. 2 Schematic representation of a spheroid model. Hypoxic nucleus containing necrotic cells, surrounded by quiescent cells and by an outer layer of actively proliferating cells

\section{D Tissue Engineered Cancer Models in Drug Discovery}

The most recent reports on 3D models applied in anti-cancer drug discovery is succinctly depicted in Table 1 and will be further discussed herein.

\section{Melanoma}

Of all types of skin cancer, melanoma is the most malignant form that presents the highest mortality rates within Europe and United States of America, which is increasing dramatically at a rate of $3-7 \%$ a year $[60,61]$. While the surgical removal of these early neoplastic cells results in $90 \%$ of cure rates, its late detection has a poor survival prognosis [62], mainly because metastatic disease derived from melanoma fails to respond to most treatments Despite the success of conventional drugs to inhibit cell growth in 2D cultures, most of them fail to do so in $3 \mathrm{D}$ tissue cultures, suggesting a potential role of the cellular microenvironment in the process of melanoma development. Indeed, the complexity of skin tissues and its cell interactions are hardly matched by $2 \mathrm{D}$ cultures.

Another crucial feature of melanomas is their ability to stimulate the formation of capillaries to supply the tumor and to penetrate the vessel walls to initiate metastatic process $[63,64]$. This emphasizes the need for models with different cell types, especially, vascularized, in order to allow the evaluation of drugs that interfere in this processes, as they may be a key target to fight it [65]. The use of animal skin models to study melanoma progression is also not an alternative, since substantial differences exist between human and animal skin. For instance, murine dermis is thin and comprises typically 3 layers of epidermis, while human possesses a thick dermis and between 6 and 10 layers of epidermis. For all these reasons, 


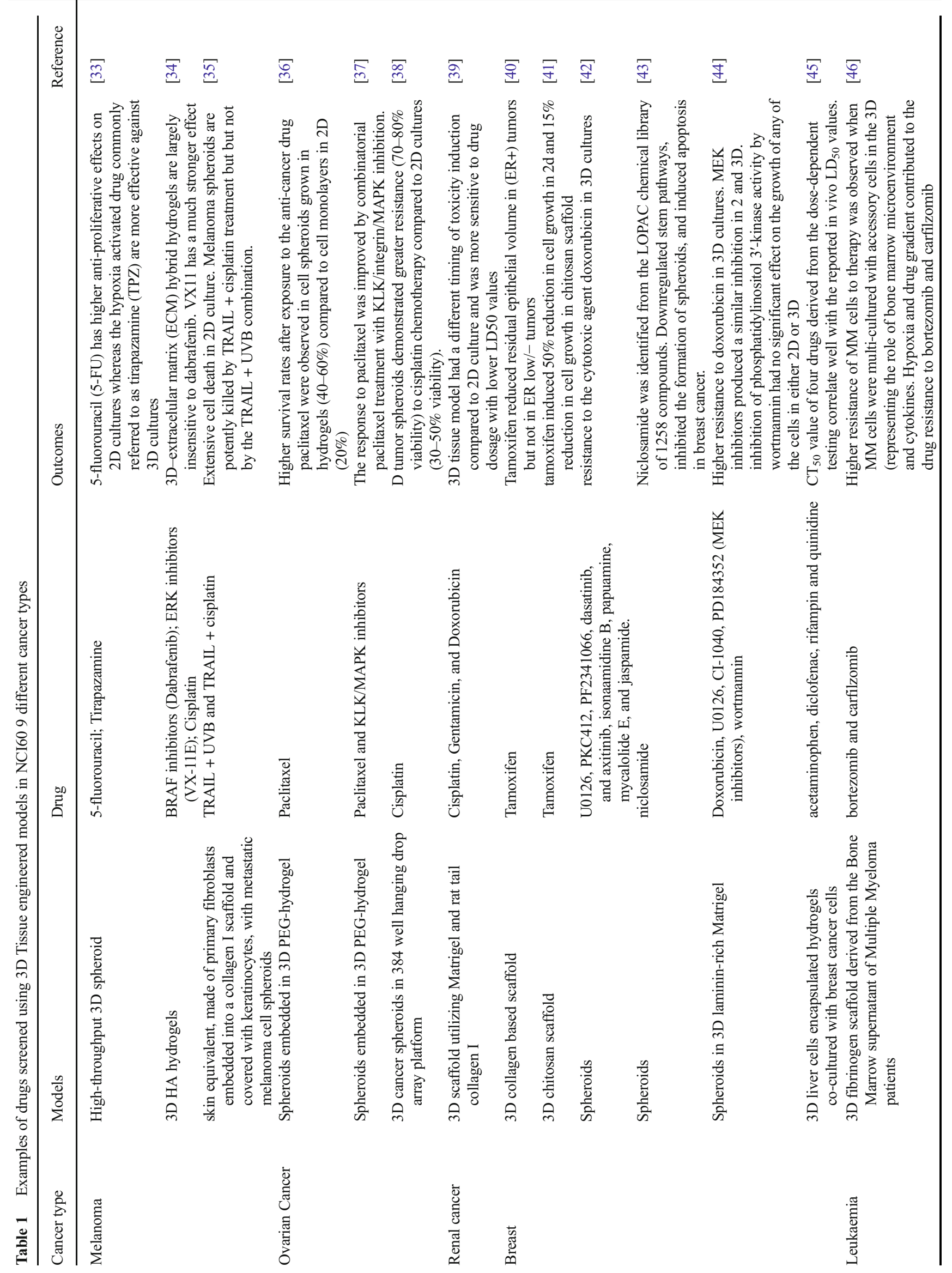




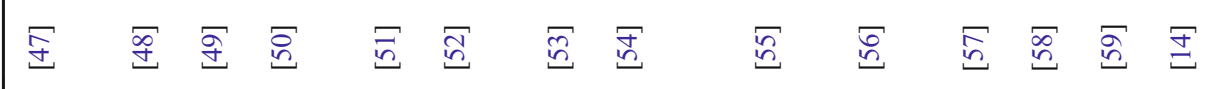

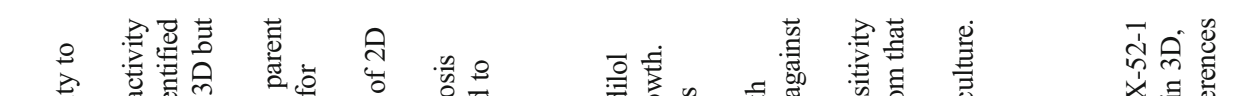

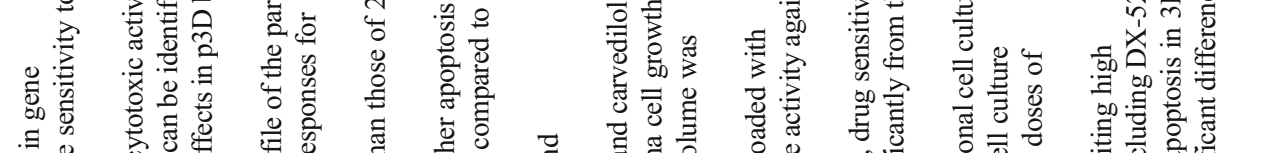

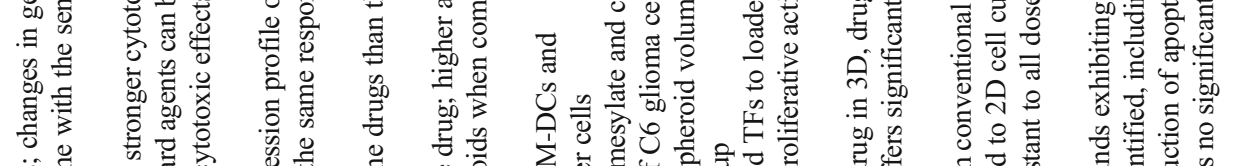

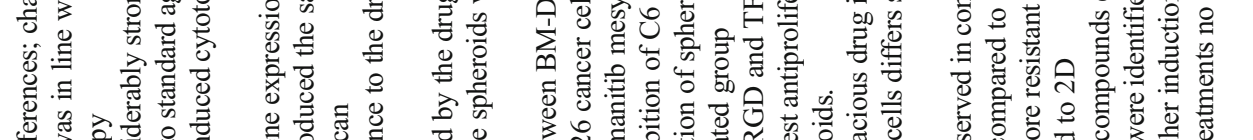

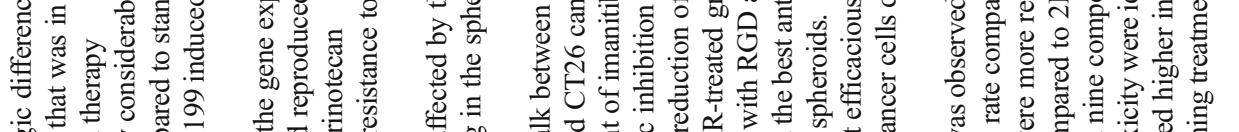

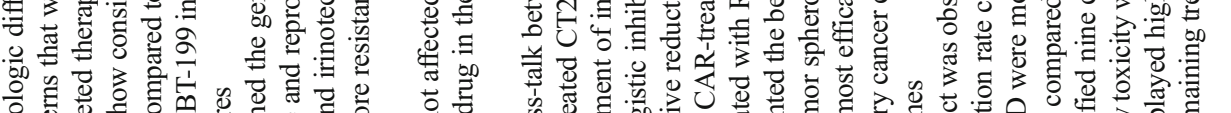

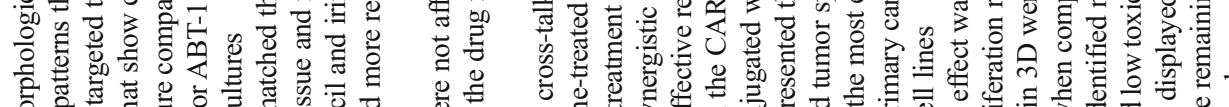

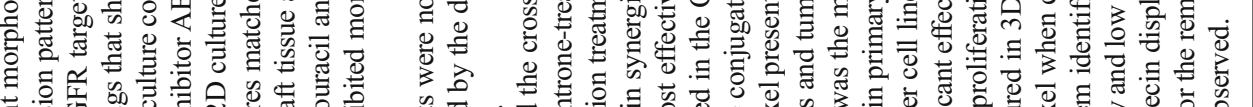

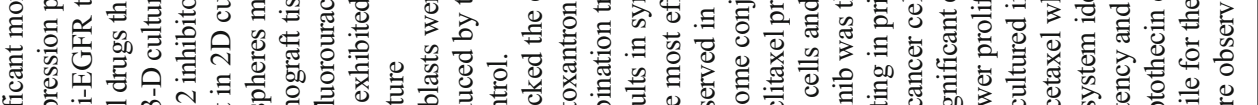

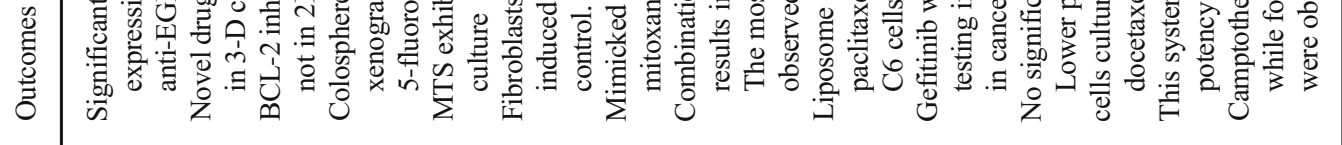

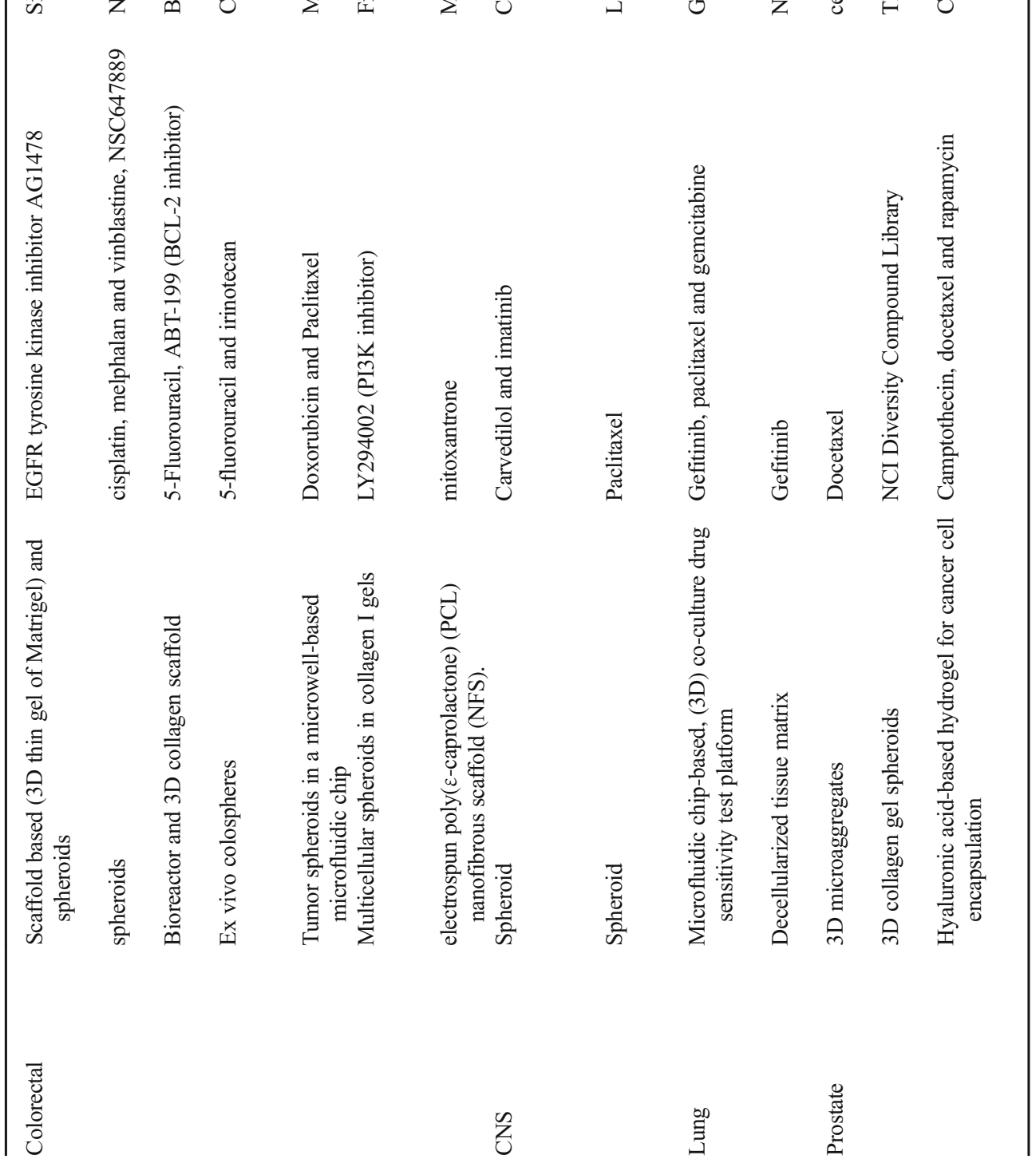


the use of 3D human skin models in the context of melanoma study is extremely relevant.

The need to complement 2D tests with $3 \mathrm{D}$ studies is well demonstrated in the case of melanoma, since A431.H9 human epithelial carcinoma cell spheroids displayed different responses to anticancer drugs, compared to 2D cultures [33]. When organized in spheroids, these cells were less sensitive to the anti-proliferative effects of the anti-cancer drug 5fluorouracil (5-FU). The hypoxia activated citotoxin, tirapazamine (TPZ), had the opposite effect, with cells displaying less resistance when grown as spheroids than in 2D monolayers [33]. These imparities are strong indicators of physiological differences associated with culture environment and the higher efficacy of TZP against cells in 3D environment might therefore be related to hypoxic core and limited oxygen diffusion characteristic of spheroids. 5-FU targets mostly cells that are actively dividing, thereby explaining the minor sensitivity of cells in spheroids. Additionally, the proportion of necrotic/proliferating cells may also explain why more sharpened differences were observed for bigger spheroids in comparison to 2D cell cultures [66]. This model advantage is the controlled size spheroids it produces, being limited by the diameter of the plateau on the bottom surface.

Another testing system, developed by Vorsmann and colleagues [35], has incorporated a skin equivalent, made of primary fibroblasts embedded into a collagen I scaffold and covered with keratinocytes, with metastatic melanoma cell spheroids (451-LU). This system allowed testing the effects of the Tumor Necrosis Factor-related Apoptosis Inducing Ligand (TRAIL), a recently developed method for inducing malignant cell apoptosis that involves the administration of compounds belonging to the Tumor Necrosis Factor (TNF) superfamily. Tests performed using this system have revealed significant differences in responses to treatments in comparison to $2 \mathrm{D}$ cultures. The combination of TRAIL with sub-lethal doses of either ultraviolet-B radiation (UVB) or cisplatin displayed similar efficacy at inducing cancer-cell death in 2D cultures. However, when they were tested using this skin-equivalent, no effect was observed in the co-treatment with TRAIL and UVB, but a potent effect was observed when treated with the TRAIL-cisplatin combination [35].

Blehm et al. [34] have tackled an essential issue in melanoma cancer: the problem of brain metastases. The team constructed platforms recreating the brain extracellular matrix (ECM) composition, mimicking the brain"s perivascular niche and hyaluronic acid (HA) rich parenchyma. The tumor growth in an HA-rich 3D matrix seeded with Fibronectin and Laminin was studied to determine the effect of cell microenvironment interactions on cell proliferation. Results revealed that ERK inhibitors (VX-11) has a much stronger effect on tumor clusters, generally affecting each cluster by inducing cell death throughout the cluster. Dabrafenib appears to be less potent, with entire clusters remaining unaffected and cell death only occurring in isolated cells at the cluster periphery. These data suggest the complexity inherent to determining drug efficacy in distinct microenvironments, and therefore the relevance of such models in drug discovery.

Such significant therapeutic differences in 3D models of human skin-melanoma, in comparison with 2D models, highlight the need to more complex representation of in vivo scenarios, in order to allow a better understanding of the biological processes underlying tumor aggressiveness, invasiveness potential and resistance to treatments.

Another emergent field in cancer research is immune oncology. This emerging field of cancer research can take advantage of three-dimensional culture technologies, which emphasizes the tumor architecture and therefore affects cancer cell-immune system interaction by regulating differentiation patterns of myeloid cells but mainly by decreasing the sensitivity of tumor cells to lymphocyte effector functions. An example was explored by Ramgolam et al., who formed melanoma spheroids neural crest cell medium confirmed that spheroids display enhanced migratory/invasive capacities [67]. In immune activation assays, spheroid cells elicited a poorer allogeneic response from immune cells and inhibited mitogen-dependent $\mathrm{T}$ cells activation and proliferation more efficiently than their adherent counterparts. Our findings reveal a novel immune-modulator function of melanoma spheroids and suggest specific roles for spheroids in invasion and in evasion of antitumor immunity [67].

\section{Ovarian Cancer}

Ovarian cancer is the eighth most common type of cancer in women [68], of which the most frequent and lethal form is the ovarian epithelial carcinoma (OEC), responsible for mortality rates around $55 \%$ Treatments for this disease typically involve surgery for removing the tumor, followed by a platinum-based chemotherapy (cisplatin or carboplatin) in combination with taxane (paclitaxel or docetaxel), which will target cell proliferation processes and inhibit cancer progression [69, 70] However, similar to other cancer types, drug efficacy results may vary significantly when cells are grown in $2 \mathrm{D}$ or in $3 \mathrm{D}$. Ovarian tumors are complex mixtures of carcinogenic, fibroblasts, mesothelial and inflammatory cells, hard to mimic in 2D cultures [71].

Despite this, the number of studies addressing this problem is fairly limited [72] and most of them use ovarian cancer cell spheroids grown in different matrices to assess chemotherapy.

The discovery of new ovarian anti-cancer drugs would benefit from the existence of 3D pre-clinical models of tumor invasion, as a typical feature of this kind of tumors is the formation of malignant spheroids that invade the peritoneal cavity at later stages of the disease [73]. This has been attempted by Loessner et al. [36], who grew ovarian cell lines (OV-MZ-6 and SKOV-3) derived from peritoneal ascites of 
EOC onto synthetic polyethylene glycol-based hydrogel matrixes, modified to contain cell integrin-binding motifs and to be degraded by cell secreted metalloproteases [36] (Fig. 3). In this $3 \mathrm{D}$ environment, cells were able to proliferate and to aggregate into spheroids, which displayed higher survival rates after exposure to paclitaxel (40-60\%) than cells grown in 2D $(20 \%)$. Furthermore, this model has later allowed to understand the role of a particular set of proteins (kallikreinrelated peptidases 4-7) in tumor invasion and revealed, for instance, that a combination of paclitaxel along with inhibition of those proteins would be more efficient in preventing metastasis [37]. Moreover, the spheroids presented sizes between 5 and $20 \mu^{3} \times 10^{4}$, mimicking the difficulty of the drug to diffuse through the cell aggregation.

Spheroids are still the easiest way to perform tests in 3D, however, they require high starting cell numbers, and are of hard long-term maintenance. Raghavan et al. [38] generate stable, uniform multicellular spheroids using a very small number of ovarian cancer cells in a novel 384-well hanging drop array platform, making it very expedite to test anti-cancer drugs. Spheroids presented sizes between 42.60 and $475.22 \times 103 \mu^{2}$ for A2780 cells and $37.24281 .01 \times 103 \mu \mathrm{m}^{2}$ for OVCAR3 cells. As expected, when compared to 2D controls, 3D tumor spheroids demonstrated greater resistance $(70-80 \%$ viability) to cisplatin chemotherapy.
Another attempt to mimic the invasion into the peritoneal cavity has been achieved by culturing primary human fibroblasts in a collagen I ECM, covered with a primary human mesothelial cell layer [71]. On top, a thin layer of collagen I was placed and covered with ovarian cancer cells, displaying histological characteristics similar to those of real patients' metastasis samples [71]. These cancer cell lines, when grown in direct contact with mesothelial cells, have their adhesion and proliferation inhibited. On the contrary, their direct contact with fibroblasts renders them more adhesive and invasive [71]. This model highlights the more relevant role of cell interaction rather than ECM in the determination of ovarian cancer invasion behavior.

Nevertheless, these complex 3D models show promising potential to contribute for a better understanding of ovarian cancer development and contribute to the discovery of new treatments.

\section{Renal Cancer}

Renal cancer is formed in kidney tissues and includes several types of carcinomas, with renal-cell carcinoma being the most frequent, responsible for about $85 \%$ of the cases [74] and corresponding to about $2 \%$ of all new cancer cases diagnosed globally [75]. Development of more efficient therapies is therefore fundamental to increase success rates, because after
Fig. 3 3D spheroid models for drug testing in cancer research. A) Melanoma spheroids respond to combination treatment in vitro. $20 \mathrm{~h}$ later, spheroids were stained for apoptosis using FITC-labeled AnnexinV (green) and propidium iodide (PI, red) and analyzed by immunofluorescence microscopy. Reprinted with permission from [35]. Copyright, 2013 Nature Publishing Group. b) Epithelial ovarian cancer cells (EOC) in 2D and $3 \mathrm{D}$ embedded within hydrogels grew as spheroids imaged by phase contrast (top panel) and confocal (bottom panel, maximal projection) microscopy. Reprinted with permission from [36]. Copyright, 2015 Elsevier

a
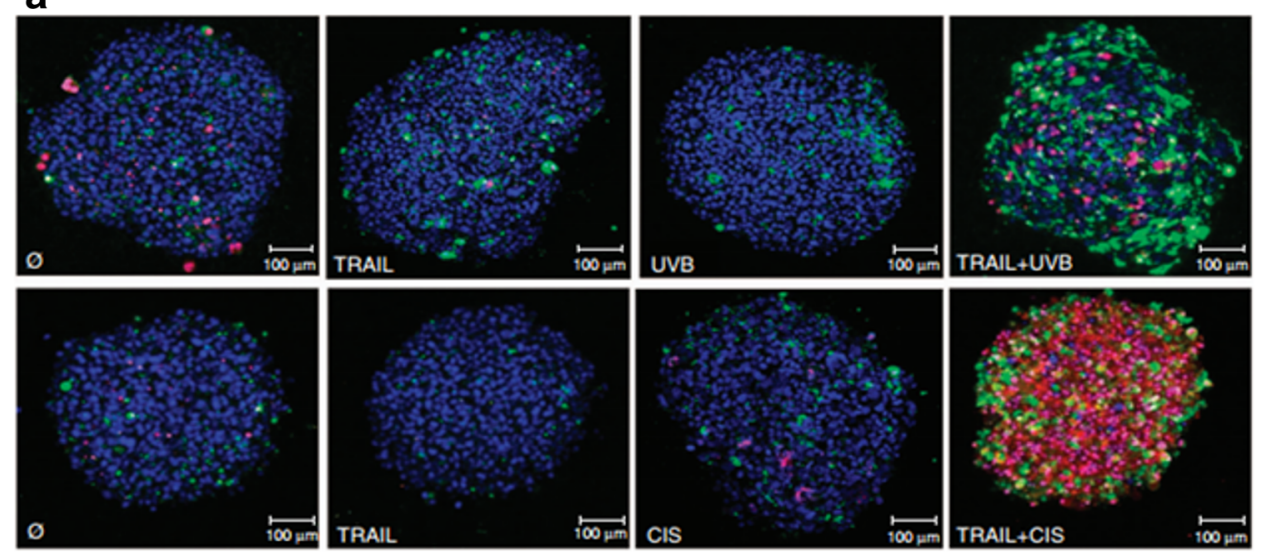

b

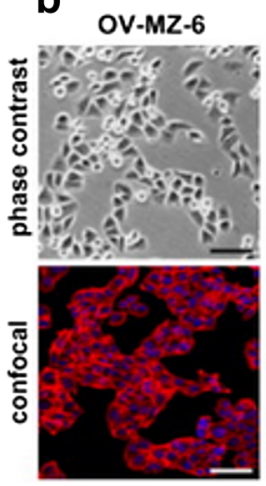

SKOV-3

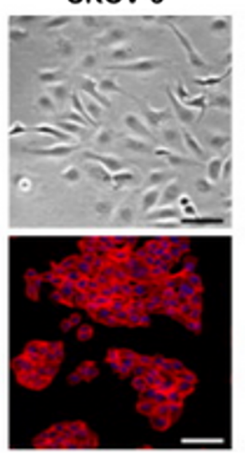

OV-MZ-6

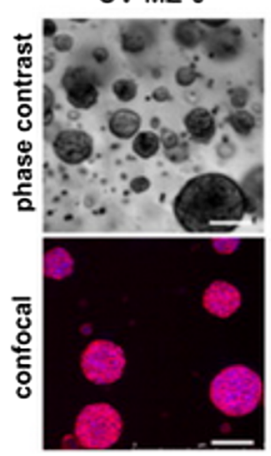

SKOV-3

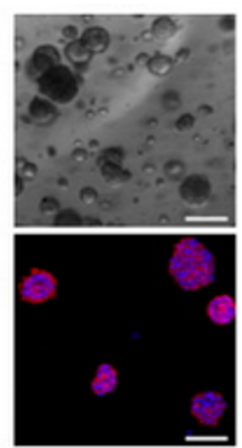


the appearance of metastasis the effective response to cytotoxic, hormonal and radio therapies is below 10\% [76-78].

Regardless the evident advantages of testing promising therapeutic drugs using in vitro 3D models, few attempts have been made to reconstruct kidney, most of them consisting in growing cells onto scaffolds, made of collagen I, Matrigel, thermo responsive polymers, hollow fibers or pre-molded biodegradable polymers $[79,80]$. Perhaps this is a result of the difficulty to mimic kidney's complex structure by in vitro models, as scaffolds hardly mimic the biochemical, spatial and vascular characteristics of the native kidney ECM [80, 81]. Kidneys physiological role in the organism (blood filtration, electrolyte, acid-base and blood pressure balance, among others) makes it an organ particularly sensitive to drug toxicity [39]. The anticancer drug cisplatin is a good example of this scenario, for despite its chemotherapeutic efficiency against several solid cancers, it is known to cause renal dysfunction in patients receiving high doses [82]. To study renal toxicity, DesRochers et al., [39] have bioengineered a 3D kidney tissue model using immortalized human renal cortical epithelial cells in a 3D Matrigel scaffold and rat tail collagen I with kidney functions similar to that found in vivo. These 3D tissues were compared to $2 \mathrm{D}$ cell cultures in terms of both acute (3 days) and chronic ( 2 weeks) toxicity induced by Cisplatin, Gentamicin, and Doxorubicin. Result show that 3D tissues were more sensitive to drug-induced toxicity and, unlike the 2D cells, were capable of being used to monitor chronic toxicity due to repeat dosing with different timing of toxicity induction. The inclusion of this tissue model in drug testing prior to the initiation of phase I clinical trials would allow for better prediction of the nephrotoxic effects of new drugs [39].

An alternative approach consisted in maintaining the structural organization of proximal tubules collected from mice by encapsulating them in commercial HA derived hydrogels [83]. Despite some oxygen diffusion problems, this model was able to maintain appropriate biomarker expressions and some biological functions up to 2 months, thus demonstrating the potential of the technique for long term drug toxicity assays. The preservation of native structural organization and cell communication appear to be an alternative to better mimic in vivo organ functions, however, its access is limited by donor availability of tissues, in case it is intended to be used with human tissues. Recently, a rudimental kidney was developed and was able to produce urine when transplanted in rat. This was achieved by seeding decellularized rat kidney scaffolds (maintaining tissue organization and structures) with kidney epithelial and endothelial cells, grown in a whole-organ bioreactor [84]. Though incipient, this novel technique appears to be promising to mimic kidney function and therefore its response to anti-cancer drugs, both in terms of therapeutic efficacy and nephrotoxicity.

Perhaps the greatest advantage in developing a kidney-like organoid able to display physiologically relevant functions, for use in anti-cancer drug development lies precisely in a better prediction of toxicity, since nephrotoxicity is strong limiting factor in drug discovery processes, namely in cancer $[82,83]$.

\section{Breast Cancer}

Breast cancer occurs in tissues of the breast and is the number one malignant tumor affecting women, although it may also affect men [3]. Breast cancer is responsible for $23 \%$ of female cancer cases worldwide and is also the leading cause of cancer deaths, accounting for $14 \%$ of total cancer deaths [68].

The interaction of cellular microenvironment and tumor development is particularly evident in breast cancer, as it has become clear that perturbations in the cell-ECM interaction are a distinguishing feature in development of such tumors [85]. Nevertheless, most drugs currently used to target this disease have been initially tested using 2D cell cultures.

Particular attention should be placed in choosing the material for growing $3 \mathrm{D}$ breast cancer cells, since this aspect alone is able to influence tissue organization [27]. Culturing normal mammary cells in collagen substrate has resulted in development of basement membrane and milk protein production [86, 87]. Their growth in a laminin-rich reconstituted basement membrane recapitulates in vivo organization of mammary acinus lumen and cells display inverted [88]. A distinctive property of malignant cells is precisely the incapacity of forming such organized structures [89]. Nevertheless, due to its biocompatibility and potential to suffer enzymatic degradation, collagen I hydrogel scaffolds have been proposed as an alternative to the creation of $3 \mathrm{D}$ in vitro tumors to study prevascularization phases [90]. Maintenance of tissue organization, cell proliferation and invasion was achieved by culturing primary breast cancer cells obtained by biopsy collection in a collagen I 3D structure [40]. This innovative technique consisted in making the hydrogels in 24-well plates and leave it for gelling at $37^{\circ} \mathrm{C}$. Tumor samples are placed on to the gelling collagen and pushed in to the center using a pipette tip. The gel is then released using a pipette tip, making it a small round shape ex vivo 3D model. This ex vivo culture method has allowed the determination of tamoxifen efficacy in a more realistic environment, taking into account cellular interactions between themselves and the surrounding stroma, as this is believed to be responsible for de novo drug resistance [40]. In this study, tamoxifen reduced residual epithelial volume in Estrogen receptor positive (ER+) tumors but not in Estrogen receptor negative ER low/-tumors, as expected. A higher resistance to tamoxifen treatment was also observed for MCF-7 breast cancer cell lines grown on a 3D chitosan scaffold [41], emphasizing the possibility of 3D cancer models being better at mimicking tissue responses to drug treatments.

The complex mechanisms underlying 3D cellular responses to drug treatments has also been pursuit. By adapting 
the reconstituted Basement Membrane culture protocol to grow MCF10 premalignant progression series of cells, $\mathrm{Li}$ and colleagues [91] managed to develop an in vitro 3D model of breast cancer progression, from normal breast epithelia to carcinomous phenotype [91]. This simple model demonstrated that cells grown in 3D were more resistant to the doxorubicin than in $2 \mathrm{D}$ cultures, thought this is more evident in normal and hyperplastic cells than carcinoma models [44]. However, the response to specific inhibitors was more variable: the exposure to MEK inhibitors, which typically leads to growth inhibition of MCF10 in 2D, encountered more resistance in both normal and hyperplastic models, while carcinoma models demonstrate higher sensitivity towards it. This higher sensitivity to MEK inhibitors (U0126 and PD184352) was also registered in MDA-MB-231 basal-subtype breast carcinoma cells when grown in 3D than 2D. In contrast, inhibition of phosphatidylinositol 3-kinase activity (wortmannin) had no effect either in 3D or 2D [44].

One of the reasons responsible for deaths associated to breast cancer is the resistance to chemotherapies and disease relapses, and breast cancer tumor recurrence has recently been attributed to a small population of cells, of stem-like characteristics, able to self-renew and to promote tumor progression [92-94]. These cells, derived from MCF7 cancer cell line, were successfully isolated using a dye-exclusion method and were further grown as spheroids, allowing to obtain a 3D spheroid tumor model with stem-like properties [43]. It was the first time that the combination of these two methods was used to select cancer stem cells. Spheroid culture was used after FACS sorting and plated in ultralow attachment plates. Subsequently, cells were observed under suspension culture conditions to detect the stem cell phenotype of tumorsphere formation [43]. Using this methodology, the authors were able to screen 1258 compounds of the LOPAC chemical library, and to unravel the potential to inhibit spheroid formation of niclosamide (a clinically approved drug used as an anthelmintic agent) thus, emphasizing the potential of this stem-like enriched spheroid formation technology in breast cancer drug discovery [43].

As technology evolves, more complex systems are continuously being developed to address drug interactions not only with target cells but also their non-target secondary effects. A new complex double system, developed by Lan et al. [45] allows the prediction of both drug efficacy and hepatotoxicity. By growing 3D cultures of liver cells encapsulated in alginate hydrogels together with a breast cancer cell line, they could assess the cytotoxicity of some commercially available drugs along with their efficacy. The test platform allowed to evaluate drug dose concentrations to predict hepatotoxicity and its effect on the breast cancer cell line. The $\mathrm{CT}_{50}$ value of four drugs (acetaminophen, diclofenac, rifampin and quinidine) derived from the dose-dependent testing correlate well with the reported in vivo $\mathrm{LD}_{50}$ values [45]. Using such systems could thus become an alternative to minimize the expensive costs associated to animal testing, estimated in $\$ 3$ billion a year [95], which not always render accurate predictions on drug efficacy and toxicity. The incorporation of breast cancer tissue models in anti-cancer drug discovery processes would undoubtedly add relevant knowledge towards the development of more suitable therapies to tackle such a complex disease.

\section{Prostate Cancer}

Prostate cancer is the second most common cancer diagnosed in men, estimated to impact about $40 \%$ of men at the age of 60 and $90 \%$ of them at the age of 90 years old $[68,96]$.

Chambers et al. [58] developed a microwell platform and surface modification protocol to enable high throughput manufacture of 3D cancer aggregates (by forced aggregation process), that perfectly allows testing of drug sensitivity and its comparison with 2D cultures. When docetaxel was tested, cells cultured in 3D were more resistant to all doses of docetaxel than those cultured in 2D. Even more interestingly, when the microaggregates are dispersed and returned to monolayer, they exhibit the same dose response to docetaxel as those cells cultured in monolayer, exacerbating the importance of tumor microenvironment and interactions [58].

It is now becoming more evident the role of the microenvironment in prostate cancer invasion, migration and metastatic behavior, as evidenced by the increase in metastatic potential and cytogenetic abnormalities when in contact with bone A metastatic 3D model recapitulated this process by growing a free-floating mouse bone sample in a bone organ culture media containing cancer prostate tumor cell lines, LnCap Clone FGC cell line and PC3 cells, using a roller tube system under hypoxia [97]. This system revealed that cancer cells preferred to colonize and proliferate on the "endosteal side" of the bone, further revealing distinct pathophysiological aspects if grown with breast or prostate cancer cell lines, thus demonstrating the amazing potential of 3D tissue engineered cancer models to study such biological events. Also, within this bone-like microenvironment, prostate cancer cells displayed morphological plasticity and altered hormonal and proteolytic responses that were not seen in traditional cell monolayer culture [97].

Another promising model to validate drugs was developed by Evensen et al. [59] that allows the segregation between compounds that inhibit cancer cell invasion and those that induce cell death. This model consists of a collagen gel spheroid-based 3-D invasion HT screening tool in an adapted 96-well plate. Cancer cells were combined with neutralized native type I collagen, dotted into the center of each well of a 96-well plate, and allowed to solidify, ultimately forming a cell-collagen dot/hemisphere with a distinct boundary and shape. Following solidification, the cell-collagen hemispheres 
were embedded within a cover-layer of neutralized type I collagen and allowed to solidify. Media supplemented with the tested drug is added to each well and the cells are allowed to invade into the surrounding [59]. This system identified nine compounds exhibiting high potency and low toxicity were identified, including DX-52-1, a compound previously reported to inhibit cell migration, a critical determinant of cancer invasion [59].

HA based hydrogels were also used to compare the efficacy of three anti-cancer drugs (camptothecin, docetaxel and rapamycin) in the treatment of bone metastatic prostate cancer, using the $\mathrm{LNCaP}$ subline $\mathrm{C} 4-2 \mathrm{~B}$, typical of bony metastases [14]. In comparison with 2D cultures, camptothecin displayed higher induction of apoptosis in 3D, while for the remaining treatments no significant differences were observed. However, a combination of docetaxel and rapamycin was more efficacious in inducing apoptosis in the HA derived hydrogel. These different results may reflect the distinct mechanisms of action and properties of the three drugs, emphasizing the importance of a proper microenvironment in anti-cancer drug efficacy assessments.

In the future, these types of 3D-tissue models may contribute to study the interaction of tumors with its microenvironment, and the way novel drugs may interfere with them. Immunotherapies have been suggested to be alternatives to conventional treatments once they become resistant. However, despite the initial success of using peripheral blood lymphocytes to target cancer cells in suspension or in $2 \mathrm{D}$ monolayer, its efficacy decreases dramatically when using 3D models [98]. A 3D platform, made of a porous chitosanalginate scaffold, has been used to study the interaction of prostate cancer cells and human peripheral blood lymphocytes highlighting its potential for use in long-term analysis and for the development of efficacious immunotherapies [98]. Despite promising, the use of 3D prostate cancer models for drug discovery appears still to be in its early steps.

\section{Leukaemia}

Leukaemia designates cancers that have their origin in tissues that form blood, such as the bone marrow, triggering the production of abnormal white blood cells and their release into the blood stream. Depending on the speed with which the disease develops, it can be classified as chronic (slow development) or acute (fast development). However, it can also be classified depending on the cell types affected, being named lymphoid, lymphocytic, or lymphoblastic when it targets lymphoid cells and myeloid, myelogenous, or myeloblastic when it targets myeloid cells (NCI n.d.). Disease prognosis depends on several factors, including the type of leukaemia [99-102].

It is becoming clearer the important role played by the bone marrow microenvironment both in the development of normal cells and in hematopoietic malignancies. It is the interaction of supporting cells (which produce growth factors, extracellular matrix molecules, cytokines and other ligands) with hematopoietic stem cells (HSCs) hematopoietic progenitor cells (HPCs) that regulates important functions such as cell proliferation, differentiation and migration [103]. It has been demonstrated that the induction of apoptosis by asparaginase (commonly used to treat pediatric acute lymphoblastic leukaemia) is blocked when malignant cells are co-cultured with stroma cells [104]. Despite this, the existence of tissue engineered models to mimic this bone marrow microenvironment and its interaction with leukaemia development is far from being achieved.

Significant differences have been observed between conventional polystyrene culture and a co-culture system in a hollow fiber bioreactor (HFBR) [105]. Erythroleukaemia K562 cells displayed higher expansion rate when cocultured in this system with supporting HS-5 stroma cells (3130 fold vs. 43 fold), thus evidencing the importance of cell-cell interactions for myelo-leukemic proliferation. With the advantage of achieving high cell densities and 3D cellular contact, the HFBR system recreates a closer mimicry of the cell-stroma microenvironment which can provide significant improvements to in vitro studies of stroma-dependent leukemic and hematopoietic cell functions and to study drug resistance.

One of the reasons suggested to explain leukaemia relapse after chemotherapy is the existence of leukaemia cell niches in the bone marrow [106]. This has been mimicked using polyurethane scaffolds coated with human mesenchymal stem cells from the bone marrow, which were further implanted in a murine host, where they developed a human bone-marrow like microenvironment, with vascularization, osteoclasts and adipocytes [106]. Injection of primary human acute myeloid leukemia cells revealed that these cells preferred the bonemarrow like scaffold to grow, rather than the host, up to three months after injection, although they were able to invade other tissues of the host after four months. The existence of more realistic tissue engineered leukaemia models could help to clarify these mechanisms and to establish more efficacious therapies. As a step further to establish 3D models of human acute myeloid leukaemia, three different sub-types of these cancer cell lines (K-562, HL60 and Kasumi-6) were grown on highly porous scaffolds made of distinct materials, namely, PLGA, PU, PMMA, PDLLA, polycaprolactone and PS [107]. Of these, only PU and PLGA allowed the long-term cellular growth (over a period of 2 months) [107]. The effect of coating of these scaffolds with collagen I or fibronectin, proteins constituting the bone marrow ECM was also assessed. However, each of the cell lines preferred a specific combination of scaffold/collagen I/fibronectin concentrations [107].

Thus, for the enumerated reasons, such model could potentially have a great impact not only to understand the biology of 
the disease progression, but also in a drug discovery perspective.

\section{Lung Cancer}

Lung cancer has its origin in lung tissues, typically in the cells lining air passages, and was responsible for $13 \%$ of all cancer cases and $18 \%$ (1.4 million) of all deaths caused by cancer in 2008 worldwide [108]. More recently, Huh et al. [109] developed a biomimetic microsystem that reconstitutes the critical functional alveolar-capillary interface of the human lung by seeding human alveolar epithelial cells and microvascular endothelial cells in the microchannels which then attached to opposite surfaces of the ECM coated membrane. Although this particular model was not tested yet for drug responses, the development of cell-based biochips that reproduce complex physiological and pathological responses could revolutionize toxicology field and development of pharmaceuticals that rely on animal testing and clinical trials [109].

In 2014, Stratmann et al. [57] established an innovative three-dimensional (3D) lung carcinoma models on a decellularized tissue matrix (porcine jejunal fragment) seeded with HCC827 or in A549 cell lines, providing a complex microenvironment for cell growth. Results show a significant decrease in proliferation upon gefitinib treatment in the $3 \mathrm{D}$ model when compared to a classic 2D cell monolayer. Once again, showing that drug treatment of cells grown in $3 \mathrm{D}$ conditions on an extracellular matrix scaffold had stronger and faster effects than in 2D cultured cells, which supports the notion that the $3 \mathrm{D}$ model is more appropriate for further drug testing.

Recently, a micro-chip co-culture scheme was developed allowing testing of several concentrations of antichemotherapeutic drugs, single or in combination, under continuous supply of fresh medium [56]. This polydimethylsiloxane microfluidic device was fabricated by replicate molding on a master onto a glass wafer and patterned by photolithography. It contains four units, each of them containing a concentration gradient generator, a reservoir and three parallel cell culture chambers, where a cell basement membrane extract mixture could be seeded to provide an adequate $3 \mathrm{D}$ environment. Important differences in cell viability responses to gefitinib, paclitaxel and gemcitabine were observed with tests performed in the human non-small cell lung cancer cell line (SPCA-1) grown in conventional monolayer, or grown in 3D in this microfluidic device, as mono-culture or co-cultured with lung fibroblast cell lines (HFL1). The most significant differences were observed for patient's cultured cells exposed to the anti-cancer agent gefitinib, when compared to SPCA-1 monoculture, thus emphasizing the drug sensitivity differences observed frequently in 2D cell lines tests and the actual tumors [56].

\section{Colorectal Cancer}

Colorectal cancer it's originated either in the colon or in rectum of the large intestine. More than $90 \%$ of colorectal malignancies are adenocarcinomas (cancerous tumor that can occur in several parts of the body, defined as neoplasia of epithelial tissue that with glandular origin, glandular characteristics, or both). Screening of altered polyps and lesions is the most effective way to target this disease, for its early detection results in 5-years survival rates over $90 \%$, compared to only $6 \%$ when detected at later stages. Treatment may include surgery, chemotherapy, radiotherapy and, less frequently, biological treatment [110].

A recent study by Mohanty and his team has demonstrated the potential of HCT-116 colon carcinoma cells grown as spheroids with $500 \mu \mathrm{m}$ of diameter to unravel novel chemotherapeutic drugs [48]. Using this model, it was evidenced a potent apoptotic effect of a new small molecule (NSC647889). Furthermore, mechanistic insights could be obtained, since resistance to treatment was observed at the inner core of the spheroids, suggesting that hypoxia and nutrient availability may limit the efficacy of the drug in an in vivo microenvironment [48].

3D spherical structures, designated as colospheres, have also been obtained spontaneously from colorectal primary tumor tissue subjected to mechanical dissociation [111]. It has been shown that this ability was cancer cell specific, because their non-tumoral cell counterparts failed to do so allowing its distinction, and was significantly correlated to the original tumor aggressiveness [111]. Furthermore, a subsequent study has shown that this colospheres had similar genetic pattern to its original tissue and were able to metastasize when implanted in mice [50]. Plus, they displayed responses to the conventional colorectal treatment drugs, 5-fluorouracil and irinotecan, identical to that of conventional xenografts [50].

In order to create an in vitro model able to recapitulate both tissue architecture and the interaction of tumor with the surrounding stromal cells, Dolzing et al. [52] went further and engineered a 3D system consisting of colon tumor cells grown first as spheroids and then co-cultured either with normal or cancer-associated fibroblasts, on a 3D collagen I scaffold. It was shown that the interaction between fibroblasts and cancer cells was responsible for the differential regulation of genes involved in important cancer associated processes, such as ECM invasion and remodeling, inflammation and angiogenesis, thus demonstrating the usefulness of such a tissue engineered model to understand cancer mechanisms [52].

Interestingly, Hirt et al. [49] have recently applied the bioreactor technology to screen antitumor compounds in colorectal cancer cells seeded on a $3 \mathrm{D}$ collagen I scaffold and compare it to traditional 2D cell cultures. Results show that perfused 3D cultures resulted in higher cell proliferation than static 3D cultures with morphology and phenotypes similar 
to xenografts. Treatment with 5-Fluorouracil (5-FU) induced apoptosis, down-regulation of anti-apoptotic genes (BCL-2, TRAF1, and c-FLIP) and decreased cell numbers in $2 \mathrm{D}$, but not in perfused cultures. Conversely, BCL-2 inhibitor ABT199 induced cytotoxic effects in 3D perfused cultures but not in $2 \mathrm{D}$ cultures. These findings reveal the importance of perfusion flow in $3 \mathrm{D}$ cultures of tumor cells to efficiently mimic functional features observed in vivo and to test anticancer compounds [49]. Following the same trend, Chen et al. ((51) developed a microfluidic platform to mimicking physiological microenvironment of solid tumor with multicellular tumor spheroids (MTS) for anticancer drug screening. The microfluidic device was fabricated from PDMS using soft lithography and rapid phototyping and has the advantage of being adapted to a microplate reader and capable to determine the cytotoxicity of the multicellular tumor spheroids. It constitutes a valuable asset for preclinical screening and evaluation procedure for anticancer agents with a wide range of tumors. The spheroids exhibited more resistance to the drugs than those of $2 \mathrm{D}$ culture based on gene expression analysis.

Kim et al. developed a 3D culture system using an electrospun poly( $\varepsilon$-caprolactone) (PCL) nanofibrous scaffold (NFS) [53] to study the immune-oncologic interactions as test the effect of mitoxantrone. Results show that when BM-DCs (bone marrow derived dendritic cells) were cocultured with mitoxantrone-treated CT26 cells (colon cancer cells) in a 3D hybrid NFS, BM-DCs sprouted cytoplasm to, migrated to, synapsed with, and engulfed mitoxantrone-treated CT26 cancer cells, which were similar to the naturally occurring crosstalk between these two types of cells. The same effect was not observed in 2D [53]. Such advanced models will represent new tools for cancer immunobiology studies and for preclinical assessment of innovative treatments.

Much work is still to be done until a 3D colorectal cancer model that fully mimics its main features can be used in drug discovery testing. Nevertheless, such model could be a fundamental tool, not only to address drug efficacy but also to increase our understanding of disease progression, from premalignant polyps and lesions to aggressive cancers.

\section{CNS Cancer}

CNS cancers are as complex and diversified as the system they affect, and therefore numerous cancer types have been defined, according to the cellular lineage in which they are originated: gliomas (it arises from glial cells, starts in the brain or spine.), medulloblastomas (the most common type of pediatric malignant primary brain tumor), meningiomas (tumors arising from the meninges, the membranous layers surrounding the central nervous system), lymphomas (lymphoma is a group of blood cell tumors that develop from lymphatic cells), pineal tumors (pineal gland tumor), acoustic neuromas (benign and usually slow-growing tumor that develops on the main nerve leading from the inner ear to the brain), haemangioblastoma (tumors of the central nervous system that originate from the vascular system usually during middle-age) and pituitary tumors (tumor in the pituitary gland) [112]. Some of them can also affect the spinal cord, in addition to the brain. Brain has specific features that make the invasion process of its tumors quite singular, such as the absence of collagen, fibronectin and laminin barriers, and its ECM is mainly composed of hyaluronan, functionalized with proteoglycan, critical for cell attachment [113, 114].

Given the described complexity, the impossibility of surgical removal in many cases and the degree of fatality associated with these types of cancer, it is clear the need of more efficient drugs to tackle it. Despite the potential of 3D tissue models as a study platform to address this need, so far few studies have been developed in this context, especially given the evidences of an association between tumor aggressiveness and interaction with ECM, at least in the case of glioblastoma [115].

Spheroid aggregates originated from U-87MG and MO59J cell lines and from glioblastoma primary culture tissue have been used to assess the efficacy of radiation therapy and have allowed the identification of some molecular pathways involved in radioresistance [116].

Other models have attempted to mimic brain ECM and its involvement in glioblastoma aggressiveness and invasion potential by using HA-based scaffolds [117, 118]. Aspects related to the role of stiffness HA hydrogels functionalized with RGD peptides in the process of gliobastoma invasion have also been addressed were studied in detail by [115]. Spheroids made of a panel of human and rat glioma cell lines were allowed to grow in different stiffness HA-hydrogels and its invasion across it assessed, showing that despite their common ability to invade the matrix, there were clear differences in invasion patterns across cell lines. As for denser hydrogels, they completely blocked spheroid invasion, though allowing spheroid growth. This model has also showed differences in cell motility pattern, when compared to both $2 \mathrm{D}$ and fibrous $3 \mathrm{D}$ collagen-based matrices, and a remarkable resemblance to that of glioma cells and neural progenitors migrating in brain slice cultures. Such model could, in the future be used to study invasion processes and their responsiveness to chemotherapeutic agents [115]. Additionally, brain tissue engineered models could be extremely helpful to understand the low efficacy of glioma treatments, since one of the reasons pointed to explain it is the existence of blood-brain and glioma-brain barriers that limit chemicals penetration in target tissues [55]. To address this issue, C6 glioma tumor cell line spheroids were grown alone or in combination with a monolayer of C6 or b.End 3 cell lines (conventionally used for the replacement of endothelial cells) to simulate penetration and efficacy of a novel drug delivery methods to target gliomas, revealing differences whether or not the additional cell barrier was present, 
thus emphasizing the need to more complex models to mimic real conditions when studying anti-cancer drugs [55]

\section{Conclusions}

Notwithstanding the historical importance of the NCI-60 cell line panel in the process of discovering novel cancer therapeutics, it is becoming ever clearer its poor efficacy at predicting animal and pre-clinical trial outcomes. This happens for reasons associated with physiological differences between natural environment and 2D cell-culture conditions. Even though, failure of predictive models happens in other clinical conditions. In the case of cancer studies this is perhaps even more striking, for it displays complex interactions with the surrounding milieu. They depend on external signals, such as connections with other cells, with ECM composition and chemical gradients (such as, oxygen or $\mathrm{pH}$. In contrast, monolayer 2D cancer cell cultures are grown in artificial plastic surfaces, rendering inappropriate topographical stimuli that culminate in different genetic patterns. Furthermore, drugs are more easily uptaken and therefore may lead to artificially higher drug efficacy rates than can never be mimicked in real conditions, with many more accessibility constraints. Monolayer cultures are usually exposed to higher levels of oxygen (typically $21 \%$ ) while oxygen tumor levels range between 1 and $5 \%$ only, being slightly more elevated in healthy tissues. 3D models for testing anti-cancer drugs could help solving some technical difficulties currently faced by common procedures, such as the poor adhesion properties of cells to their substrate or the difficulty to maintain their biological in vivo function. However such progress in research also brings with new challenges. 3D culture methods represent and expensive approach and still require solid expertise in handling and optimization to build good in vitro models, especially for less trained researchers. In addition, utilizing 3D cultures with standard cell analysis methods, which were originally developed to support 2D cultures, can be challenging. There is a need to develop microfluidic devices to achieve high detection efficiency that could make use of the multiple biochemical and physical cues that are distinctive in cancer [26].

Perhaps the gold standard in drug discovery is the use of in vivo animal testing, especially in rodents. However, they present some known limitations, such as differences in immune systems and genetic toolkit, which can explain the reduced predictability in some cases. Adding another dimension to cancer studies would neither replace existing 2D cell cultures nor animal testing, but it could definitively contribute to improve the predictability of efficacy and toxicity assessments, since 3D models usually display genetic expression and drug responses behaviors more similar to the actual tumors than their $2 \mathrm{D}$ cultures.
Tissue engineering can be the key for the development of more realistic 3D cancer models. By combining different extracellular matrix biomaterials with different cell types (including co-cultures) and different growth factors, it will be possible to mimic more realistically the complex structural organization of in vivo tumors. Ultimately, this will allow a better understanding of the role of particular set of proteins and/or cell interactions in tumor invasion behavior and hopefully enable a more efficient design of anti-cancer drugs and treatments.

It is clear that much of the success in anti-cancer drug discovery depends on using representative models, but even though many studies have demonstrated differences in drug sensitivity between $2 \mathrm{D}$ and 3D models, a demonstration of a better prediction of clinical outcomes is still missing. Yet, considering the multitude of potential mechanisms targeted by anti-cancer therapies, it is more likely that testing performed in $3 \mathrm{D}$ heterotypic cultures provides more meaningful predictions for successful in vivo outcomes than $2 \mathrm{D}$ cultures. The pharmaceutical industry is facing unprecedented challenges owing to rising costs and the declining efficiency of drug research and development, mainly due to the poor predictive capacity of current preclinical models. Pharmaceutical companies are also beginning to create partnerships with academic institutions to jointly discover 3D models and organon-a-chip devices technology and to position themselves at the vanguard of the field. We are also witnessing a growing number of commercial devices suitable for drug screening, such as MIMETAS $®$, Cellix $®$, 3D BioMatrix $®$, which can be applied in cancer research. However, there are many parameters associated with this kind of research. Between the choice of polymers, cell types, concentrations and densities, biomolecules, bioreactors and biochips, regulatory agencies are understandably cautious in accepting new sources of data as justification for human trials. Therefore, these models will have to endure stringent validation to serve as formal preclinical tests.

It is necessary however, that efforts are put into standardization of such procedures so that the developed 3D model can be used in routine diagnostic testing, as a bridge to fill in the gap that currently exists between $2 \mathrm{D}$ and animal testing. Clearly, there is still a long road ahead but there is little doubt that this is the right direction to pursue in the search for novel and more efficacious treatments to defeat one of the most frequent and pernicious diseases of our days, cancer.

Acknowledgements This article is a result of the project FROnTHERA (NORTE-01-0145-FEDER-000023), supported by Norte Portugal Regional Operational Programme (NORTE 2020), under the PORTUGAL 2020 Partnership Agreement, through the European Regional Development Fund (ERDF). This articlewas also supported by the EU Framework Programme for Research and Innovation HORIZON 2020 (H2020) under grant agreement $n^{\circ} 668983-$ FoReCaST. FCT distinction attributed to Joaquim M. Oliveira (IF/ 
00423/2012) and Vitor M. Correlo (IF/01214/2014) under the Investigator FCT program is also greatly acknowledged.

\section{References}

1. Calvert, H. (2014). Cancer Drug Design and Discovery. In S. Neidle (Ed.), Cancer Drug Design and Discovery (Second ed., pp. xi-xiii). San Diego: Academic Press.

2. Alemany-Ribes, M., \& Semino, C. E. (2014). Bioengineering 3D environments for cancer models. Advanced Drug Delivery Reviews, 79-80, 40-49.

3. WHO. Essential drugs for cancer chemotherapy. (1994). WHO consultation. Bulletin of the World Health Organization, 72, 693-698.

4. Burger, A., \& Fiebig, H.-H. (2004). Preclinical screening for new anticancer agents. In H. McLeod (Ed.), Figg W (pp. 29-44). Handbook of Anticancer Pharmacokinetics and Pharmacodynamics: Humana Press.

5. Burger, A. M., \& Fiebig, H.-H. (2014). Preclinical screening for new anticancer agents. Handbook of Anticancer Pharmacokinetics and Pharmacodynamics: Springer, 23-38.

6. Nietzer, S., Dandekar, G., Walles, H., Wasik, M. (2012). Three dimensional tissue models for research in oncology: INTECH open access Publisher.

7. Hutchinson, L., \& Kirk, R. (2011). High drug attrition rates-where are we going wrong? Nature Reviews. Clinical Oncology, 8, 189190.

8. Blatt, N. L., Mingaleeva, R. N., Solovieva, V. V., Khaiboullina, S. F., Lombardi, V. C., \& Rizvanov, A. A. (2013). Application of cell and tissue culture systems for anticancer drug screening. World Applied Sciences Journal, 23, 315-325.

9. Shoemaker, R. H. (2006). The NCI60 human tumour cell line anticancer drug screen. Nature Reviews Cancer, 6, 813-823.

10. Ross, D. T., Scherf, U., Eisen, M. B., et al. (2000). Systematic variation in gene expression patterns in human cancer cell lines. Nature Genetics, 24, 227-235.

11. Van Staveren W, Solís DW, Hebrant A, Detours V, Dumont JE, Maenhaut C. (2009). Human cancer cell lines: Experimental models for cancer cells in situ? For cancer stem cells? Biochimica et Biophysica Acta (BBA)-Reviews on Cancer, 1795, 92-103.

12. Ridky, T. W., Chow, J. M., Wong, D. J., \& Khavari, P. A. (2010). Invasive three-dimensional organotypic neoplasia from multiple normal human epithelia. Nature Medicine, $16,1450-1455$.

13. Feder-Mengus, C., Ghosh, S., Reschner, A., Martin, I., \& Spagnoli, G. C. (2008). New dimensions in tumor immunology: what does 3D culture reveal? Trends in Molecular Medicine, 14, 333-340.

14. Gurski, L. A., Jha, A. K., Zhang, C., Jia, X., \& Farach-Carson, M. C. (2009). Hyaluronic acid-based hydrogels as 3D matrices for in vitro evaluation of chemotherapeutic drugs using poorly adherent prostate cancer cells. Biomaterials, 30, 6076-6085.

15. Gorlach, A., Herter, P., Hentschel, H., Frosch, P. J., \& Acker, H. (1994). Effects of nIFN beta and rIFN gamma on growth and morphology of two human melanoma cell lines: comparison between two- and three-dimensional culture. International Journal of Cancer:Journal International du Cancer, 56, 249-254.

16. Chignola, R., Schenetti, A., Andrighetto, G., et al. (2000). Forecasting the growth of multicell tumour spheroids: implications for the dynamic growth of solid tumours. Cell Proliferation, 33, 219-229.
17. Wozniak, M. A., Modzelewska, K., Kwong, L., \& Keely, P. J. (2004). Focal adhesion regulation of cell behavior. Biochimica et Biophysica Acta, 1692, 103-119.

18. Cheema, U., Brown, R. A., Alp, B., \& MacRobert, A. J. (2008). Spatially defined oxygen gradients and vascular endothelial growth factor expression in an engineered 3D cell model. Cellular and Molecular Life Sciences: CMLS, 65, 177-186.

19. Yamazaki, D., Kurisu, S., \& Takenawa, T. (2009). Involvement of Rac and rho signaling in cancer cell motility in 3D substrates. Oncogene, 28, 1570-1583.

20. David, L., Dulong, V., Le Cerf, D., Cazin, L., Lamacz, M., \& Vannier, J. P. (2008). Hyaluronan hydrogel: an appropriate threedimensional model for evaluation of anticancer drug sensitivity. Acta Biomaterialia, 4, 256-263.

21. Horning, J. L., Sahoo, S. K., Vijayaraghavalu, S., et al. (2008). 3 D tumor model for in vitro evaluation of anticancer drugs. Molecular Pharmaceutics, 5, 849-862.

22. Serebriiskii, I., Castello-Cros, R., Lamb, A., Golemis, E. A., \& Cukierman, E. (2008). Fibroblast-derived 3D matrix differentially regulates the growth and drug-responsiveness of human cancer cells. Matrix biology : journal of the International Society for Matrix Biology, 27, 573-585.

23. Wenzel, C., Riefke, B., Grundemann, S., et al. (2014). 3D highcontent screening for the identification of compounds that target cells in dormant tumor spheroid regions. Experimental Cell Research, 323, 131-143.

24. Ratner B HA, Schoen F, Lemons J. (2004). Biomaterials science in: Elsevier Academic Press, ed. 3rd edition ed. San Diego.

25. Rimann, M., \& Graf-Hausner, U. (2012). Synthetic 3D multicellular systems for drug development. Current Opinion in Biotechnology, 23, 803-809.

26. Carvalho, M. R., Lima, D., Reis, R. L., Correlo, V. M., \& Oliveira, J. M. (2015). Evaluating biomaterial- and microfluidic-based 3D tumor models. Trends in Biotechnology, 33, 667-678.

27. Elliott, N. T., \& Yuan, F. (2011). A review of three-dimensional in vitro tissue models for drug discovery and transport studies. Journal of Pharmaceutical Sciences, 100, 59-74.

28. Peck, Y., \& Wang, D. A. (2013). Three-dimensionally engineered biomimetic tissue models for in vitro drug evaluation: delivery, efficacy and toxicity. Expert Opinion on Drug Delivery, 10, 369-383.

29. Tredan, O., Galmarini, C. M., Patel, K., \& Tannock, I. F. (2007). Drug resistance and the solid tumor microenvironment. Journal of the National Cancer Institute, 99, 1441-1454.

30. Nyga, A., Cheema, U., \& Loizidou, M. (2011). 3D tumour models: novel in vitro approaches to cancer studies. Journal of cell communication and signaling, 5, 239-248.

31. Fennema, E., Rivron, N., Rouwkema, J., van Blitterswijk, C., \& de Boer, J. (2013). Spheroid culture as a tool for creating 3D complex tissues. Trends in Biotechnology, 31, 108-115.

32. Chen, Y. A., King, A. D., Shih, H. C., et al. (2011). Generation of oxygen gradients in microfluidic devices for cell culture using spatially confined chemical reactions. Lab on a Chip, 11, 36263633.

33. Tung, Y. C., Hsiao, A. Y., Allen, S. G., Torisawa, Y. S., Ho, M., \& Takayama, S. (2011). High-throughput 3D spheroid culture and drug testing using a 384 hanging drop array. The Analyst, 136, 473-478.

34. Blehm, B. H., Jiang, N., Kotobuki, Y., \& Tanner, K. (2015). Deconstructing the role of the ECM microenvironment on drug efficacy targeting MAPK signaling in a pre-clinical platform for cutaneous melanoma. Biomaterials, 56, 129-139.

35. Vorsmann, H., Groeber, F., Walles, H., et al. (2013). Development of a human three-dimensional organotypic skin-melanoma spheroid model for in vitro drug testing. Cell Death \& Disease, 4, e719. 
36. Loessner, D., Stok, K. S., Lutolf, M. P., Hutmacher, D. W., Clements, J. A., \& Rizzi, S. C. (2010). Bioengineered 3D platform to explore cell-ECM interactions and drug resistance of epithelial ovarian cancer cells. Biomaterials, 31, 8494-8506.

37. Loessner, D., Rizzi, S. C., Stok, K. S., et al. (2013). A bioengineered 3D ovarian cancer model for the assessment of peptidase-mediated enhancement of spheroid growth and intraperitoneal spread. Biomaterials, 34, 7389-7400.

38. Raghavan, S., Ward, M. R., Rowley, K. R., et al. (2015). Formation of stable small cell number three-dimensional ovarian cancer spheroids using hanging drop arrays for preclinical drug sensitivity assays. Gynecologic Oncology, 138, 181-189.

39. DesRochers, T. M., Suter, L., Roth, A., \& Kaplan, D. L. (2013). Bioengineered 3D human kidney tissue, a platform for the determination of nephrotoxicity. PloS One, 8, e59219.

40. Leeper, A. D., Farrell, J., Williams, L. J., et al. (2012). Determining tamoxifen sensitivity using primary breast cancer tissue in collagen-based three-dimensional culture. Biomaterials, $33,907-915$

41. Dhiman, H. K., Ray, A. R., \& Panda, A. K. (2005). Threedimensional chitosan scaffold-based MCF-7 cell culture for the determination of the cytotoxicity of tamoxifen. Biomaterials, 26, 979-986.

42. Li, Q., Chen, C., Kapadia, A., et al. (2011). 3D models of epithelial-mesenchymal transition in breast cancer metastasis: high-throughput screening assay development, validation, and pilot screen. Journal of Biomolecular Screening, 16, 141-154.

43. Wang, Y. C., Chao, T. K., Chang, C. C., Yo, Y. T., Yu, M. H., \& Lai, H. C. (2013). Drug screening identifies niclosamide as an inhibitor of breast cancer stem-like cells. PloS One, 8, e 74538.

44. Li, Q., Chow, A. B., \& Mattingly, R. R. (2010). Threedimensional overlay culture models of human breast cancer reveal a critical sensitivity to mitogen-activated protein kinase kinase inhibitors. The Journal of Pharmacology and Experimental Therapeutics, 332, 821-828.

45. Lan, S. F., \& Starly, B. (2011). Alginate based 3D hydrogels as an in vitro co-culture model platform for the toxicity screening of new chemical entities. Toxicology and Applied Pharmacology, $256,62-72$.

46. de la Puente, P., Muz, B., Gilson, R. C., et al. (2015). 3D tissueengineered bone marrow as a novel model to study pathophysiology and drug resistance in multiple myeloma. Biomaterials, 73, $70-84$.

47. Luca, A. C., Mersch, S., Deenen, R., et al. (2013). Impact of the 3D microenvironment on phenotype, gene expression, and EGFR inhibition of colorectal cancer cell lines. PloS One, 8, e59689.

48. Mohanty, C., Fayad, W., Olofsson, M. H., et al. (2013). Massive induction of apoptosis of multicellular tumor spheroids by a novel compound with a calmodulin inhibitor-like mechanism. Journal of Cancer Therapeutics and Research, 2.

49. Hirt, C., Papadimitropoulos, A., Muraro, M. G., et al. (2015). Bioreactor-engineered cancer tissue-like structures mimic phenotypes, gene expression profiles and drug resistance patterns observed "in vivo". Biomaterials, 62, 138-146.

50. Weiswald, L. B., Richon, S., Massonnet, G., et al. (2013). A shortterm colorectal cancer sphere culture as a relevant tool for human cancer biology investigation. British Journal of Cancer, 108, 1720-1731.

51. Chen, Y., Gao, D., Liu, H., Lin, S., \& Jiang, Y. (2015). Drug cytotoxicity and signaling pathway analysis with threedimensional tumor spheroids in a microwell-based microfluidic chip for drug screening. Analytica Chimica Acta, 898, 85-92.

52. Dolznig, H., Rupp, C., Puri, C., et al. (2011). Modeling colon adenocarcinomas in vitro a $3 \mathrm{D}$ co-culture system induces cancer-relevant pathways upon tumor cell and stromal fibroblast interaction. The American Journal of Pathology, 179, 487-501.

53. Kim, T. E., Kim, C. G., Kim, J. S., et al. (2016). Threedimensional culture and interaction of cancer cells and dendritic cells in an electrospun nano-submicron hybrid fibrous scaffold. International Journal of Nanomedicine, 11, 823-835.

54. Erguven, M., Yazihan, N., Aktas, E., et al. (2010). Carvedilol in glioma treatment alone and with imatinib in vitro. International Journal of Oncology, 36, 857-866.

55. Qin, L., Wang, C. Z., Fan, H. J., et al. (2014). A dual-targeting liposome conjugated with transferrin and arginine-glycineaspartic acid peptide for glioma-targeting therapy. Oncology Letters, 8, 2000-2006.

56. Xu, Z., Gao, Y., Hao, Y., et al. (2013). Application of a microfluidic chip-based 3D co-culture to test drug sensitivity for individualized treatment of lung cancer. Biomaterials, 34, 4109 4117.

57. Stratmann, A. T., Fecher, D., Wangorsch, G., et al. (2014). Establishment of a human 3D lung cancer model based on a biological tissue matrix combined with a Boolean in silico model. Molecular Oncology, 8, 351-365.

58. Chambers, K. F., Mosaad, E. M., Russell, P. J., Clements, J. A., \& Doran, M. R. (2015). Correction: 3D cultures of prostate cancer cells cultured in a novel high-throughput culture platform are more resistant to chemotherapeutics compared to cells cultured in monolayer. PloS One, 10, e0125641.

59. Evensen, N. A., Li, J., Yang, J., et al. (2013). Development of a high-throughput three-dimensional invasion assay for anti-cancer drug discovery. PloS One, 8, e82811.

60. Lui, P., Cashin, R., Machado, M., Hemels, M., Corey-Lisle, P. K., \& Einarson, T. R. (2007). Treatments for metastatic melanoma: synthesis of evidence from randomized trials. Cancer Treatment Reviews, 33, 665-680.

61. Kyrgidis, A., Valasidis, A., Bourlidou, E., \& Andreadis, C. (2011). Better targeting melanoma: options beyond surgery and conventional chemotherapy. Recent Patents on Endocrine, Metabolic \& Immune Drug Discovery, 5, 147-159.

62. Spagnolo, F., \& Queirolo, P. (2012). Upcoming strategies for the treatment of metastatic melanoma. Archives of Dermatological Research, 304, 177-184.

63. Folkman, J. (1990). What is the evidence that tumors are angiogenesis dependent? Journal of the National Cancer Institute, 82, 4-6.

64. Liotta, L. A., Kleinerman, J., \& Saidel, G. M. (1974). Quantitative relationships of intravascular tumor cells, tumor vessels, and pulmonary metastases following tumor implantation. Cancer Research, 34, 997-1004.

65. Groeber, F., Holeiter, M., Hampel, M., Hinderer, S., \& SchenkeLayland, K. (2011). Skin tissue engineering-in vivo and in vitro applications. Advanced Drug Delivery Reviews, 63, 352-366.

66. Mehta, G., Hsiao, A. Y., Ingram, M., Luker, G. D., \& Takayama, S. (2012). Opportunities and challenges for use of tumor spheroids as models to test drug delivery and efficacy. Journal of Controlled Release: Official Journal of the Controlled Release Society, 164, 192-204.

67. Ramgolam, K., Lauriol, J., Lalou, C., et al. (2011). Melanoma spheroids grown under neural crest cell conditions are highly plastic migratory/invasive tumor cells endowed with immunomodulator function. PloS One, 6, e18784.

68. Jemal, A., Bray, F., Center, M. M., Ferlay, J., Ward, E., \& Forman, D. (2011). Global cancer statistics. CA: A Cancer Journal for Clinicians, 61, 69-90.

69. Piccart, M., Lamb, H., \& Vermorken, J. B. (2001). Current and future potential roles of the platinum drugs in the treatment of ovarian cancer. Annals of Oncology, 12, 1195-1203. 
70. Guppy, A. E., Nathan, P. D., \& Rustin, G. J. (2005). Epithelial ovarian cancer: a review of current management. Clinical Oncology, 17, 399-411.

71. Kenny, H. A., Dogan, S., Zillhardt, M., et al. (2009). Organotypic models of metastasis: a three-dimensional culture mimicking the human peritoneum and omentum for the study of the early steps of ovarian cancer metastasis. Cancer Treatment and Research, 149, 335-351.

72. Grun, B., Benjamin, E., Sinclair, J., et al. (2009). Threedimensional in vitro cell biology models of ovarian and endometrial cancer. Cell Proliferation, 42, 219-228.

73. Shield, K., Ackland, M. L., Ahmed, N., \& Rice, G. E. (2009). Multicellular spheroids in ovarian cancer metastases: biology and pathology. Gynecologic Oncology, 113, 143-148.

74. Cohen, H. T., \& McGovern, F. J. (2005). Renal-cell carcinoma. New England Journal of Medicine, 353, 2477-2490.

75. McLaughlin, J. K., Lipworth, L., Tarone, R. E. (2006). Epidemiologic aspects of renal cell carcinoma. Seminars in oncology; Elsevier. p. 527-33.

76. Harris, D. T. (1983). Hormonal therapy and chemotherapy of renal-cell carcinoma. Seminars in Oncology, 10, 422430.

77. Yagoda, A., \& Bander, N. H. (1989). Failure of cytotoxic chemotherapy, 1983-1988, and the emerging role of monoclonal antibodies for renal cancer. Urologia Internationalis, 44, 338-345.

78. Yang, J. C., \& Childs, R. (2006). Immunotherapy for renal cell cancer. Journal of Clinical Oncology, 24, 5576-5583.

79. Wang, P.-C., \& Takezawa, T. (2005). Reconstruction of renal glomerular tissue using collagen vitrigel scaffold. Journal of Bioscience and Bioengineering, 99, 529-540.

80. Perin, L., Da Sacco, S., \& De Filippo, R. E. (2011). Regenerative medicine of the kidney. Advanced Drug Delivery Reviews, 63, 379-387.

81. Peloso, A., Katari, R., Patel, T., et al. (2013). Considerations on the development of a model of kidney bioengineering and regeneration in rats. Expert Review of Medical Devices, 10, 597-601.

82. Yao, X., Panichpisal, K., Kurtzman, N., \& Nugent, K. (2007). Cisplatin nephrotoxicity: a review. The American Journal of the Medical Sciences, 334, 115-124.

83. Astashkina, A. I., Mann, B. K., Prestwich, G. D., \& Grainger, D. W. (2012). A 3-D organoid kidney culture model engineered for high-throughput nephrotoxicity assays. Biomaterials, 33, 47004711.

84. Song, J. J., Guyette, J. P., Gilpin, S. E., Gonzalez, G., Vacanti, J. P., \& Ott, H. C. (2013). Regeneration and experimental orthotopic transplantation of a bioengineered kidney. Nature Medicine, 19, 646-651.

85. Bissell, M. J. (2007). Modelling molecular mechanisms of breast cancer and invasion: lessons from the normal gland. Biochemical Society Transactions, 35, 18-22.

86. Lee, E., Parry, G., \& Bissell, M. J. (1984). Modulation of secreted proteins of mouse mammary epithelial cells by the collagenous substrata. The Journal of Cell Biology, 98, 146-155.

87. Streuli, C. H., \& Bissell, M. J. (1990). Expression of extracellular matrix components is regulated by substratum. The Journal of Cell Biology, 110, 1405-1415.

88. Schmeichel, K. L., \& Bissell, M. J. (2003). Modeling tissuespecific signaling and organ function in three dimensions. Journal of Cell Science, 116, 2377-2388.

89. Petersen, O. W., Ronnov-Jessen, L., Howlett, A. R., \& Bissell, M. J. (1992). Interaction with basement membrane serves to rapidly distinguish growth and differentiation pattern of normal and malignant human breast epithelial cells. Proceedings of the National
Academy of Sciences of the United States of America, 89, 9064 9068.

90. Szot, C. S., Buchanan, C. F., Freeman, J. W., \& Rylander, M. N. (2011). 3D in vitro bioengineered tumors based on collagen I hydrogels. Biomaterials, 32, 7905-7912.

91. Li, Q., Mullins, S. R., Sloane, B. F., \& Mattingly, R. R. (2008). p21-activated kinase 1 coordinates aberrant cell survival and pericellular proteolysis in a three-dimensional culture model for premalignant progression of human breast cancer. Neoplasia, 10, 314-329.

92. Bonnet, D., \& Dick, J. E. (1997). Human acute myeloid leukemia is organized as a hierarchy that originates from a primitive hematopoietic cell. Nature Medicine, 3, 730-737.

93. Reya, T., Morrison, S. J., Clarke, M. F., \& Weissman, I. L. (2001). Stem cells, cancer, and cancer stem cells. Nature, 414, 105-111.

94. Clarke, M. F., \& Fuller, M. (2006). Stem cells and cancer: two faces of eve. Cell, 124, 1111-1115.

95. van Vliet, E. (2011). Current standing and future prospects for the technologies proposed to transform toxicity testing in the twentyfirst century. ALTEX, 28, 17-44.

96. Hodge, A. C., Fenster, A., Downey, D. B., \& Ladak, H. M. (2006). Prostate boundary segmentation from ultrasound images using 2D active shape models: optimisation and extension to 3D. Computer Methods and Programs in Biomedicine, 84, 99-113.

97. Curtin, P., Youm, H., \& Salih, E. (2012). Threedimensional cancer-bone metastasis model using ex-vivo co-cultures of live calvarial bones and cancer cells. Biomaterials, 33, 1065-1078.

98. Florczyk, S. J., Liu, G., Kievit, F. M., Lewis, A. M., Wu, J. D., \& Zhang, M. (2012). 3D porous chitosan-alginate scaffolds: a new matrix for studying prostate cancer cell-lymphocyte interactions in vitro. Advanced Healthcare Materials, 1, 590-599.

99. Bakker, E., Qattan, M., Mutti, L., Demonacos, C., KrsticDemonacos, M. (2015). The role of microenvironment and immunity in drug response in leukemia. Biochimica et Biophysica Acta (BBA)-Molecular Cell Research.

100. Moses, B.S., Slone, W.L., Thomas, P., et al. (2015). Bone marrow microenvironment modulation of acute lymphoblastic leukemia phenotype. Experimental hematology.

101. Qureshi, A. K., \& Hall, G. W. (2013). Leukaemias: a review. Paediatrics \& Child Health, 23, 461-466.

102. Bakker, E., Qattan, M., Mutti, L., Demonacos, C., KrsticDemonacos, M. (2016). The role of microenvironment and immunity in drug response in leukemia. Biochimica et Biophysica Acta (BBA) - Molecular Cell Research 1863, 414-426.

103. Sison, E.A.R., \& Brown, P. (2011). The bone marrow microenvironment and leukemia: Biology and therapeutic targeting. Expert review of hematology, 4, 271-283

104. Iwamoto, S., Mihara, K., Downing, J. R., Pui, C.-H., \& Campana, D. (2007). Mesenchymal cells regulate the response of acute lymphoblastic leukemia cells to asparaginase. Journal of Clinical Investigation, 117, 1049.

105. Usuludin, S. B., Cao, X., \& Lim, M. (2012). Co-culture of stromal and erythroleukemia cells in a perfused hollow fiber bioreactor system as an in vitro bone marrow model for myeloid leukemia. Biotechnology and Bioengineering, 109, 1248-1258.

106. Vaiselbuh, S. R., Edelman, M., Lipton, J. M., \& Liu, J. M. (2010). Ectopic human mesenchymal stem cell-coated scaffolds in NOD/ SCID mice: an in vivo model of the leukemia niche. Tissue engineering Part C, Methods, 16, 1523-1531.

107. Blanco, T. M., Mantalaris, A., Bismarck, A., \& Panoskaltsis, N. (2010). The development of a three-dimensional scaffold for ex vivo biomimicry of human acute myeloid leukaemia. Biomaterials, 31, 2243-2251.

108. Marko-Varga, G., Fehniger, T. E., Rezeli, M., Döme, B., Laurell, T., \& Végvári, Á. (2011). Drug localization in different lung 
cancer phenotypes by MALDI mass spectrometry imaging. Journal of Proteomics, 74, 982-992.

109. Huh, D., Matthews, B. D., Mammoto, A., Montoya-Zavala, M., Hsin, H. Y., \& Ingber, D. E. (2010). Reconstituting organ-level lung functions on a chip. Science, 328, 1662-1668.

110. Fleming, M., Ravula, S., Tatishchev, S. F., \& Wang, H. L. (2012). Colorectal carcinoma: pathologic aspects. Journal of gastrointestinal oncology, 3, 153-173.

111. Weiswald, L. B., Richon, S., Validire, P., et al. (2009). Newly characterised ex vivo colospheres as a three-dimensional colon cancer cell model of tumour aggressiveness. British Journal of Cancer, 101, 473-482.

112. Hopkins, A. M., DeSimone, E., Chwalek, K., \& Kaplan, D. L. (2015). 3D in vitro modeling of the central nervous system. Progress in Neurobiology, 125, 1-25.

113. Shoffstall, A. J., Taylor, D. M., \& Lavik, E. B. (2012). Engineering therapies in the CNS: what works and what can be translated. Neuroscience Letters, 519, 147-154.
114. Gladson, C. L. (1999). The extracellular matrix of gliomas: modulation of cell function. Journal of Neuropathology and Experimental Neurology, 58, 1029-1040.

115. Ananthanarayanan, B., Kim, Y., \& Kumar, S. (2011). Elucidating the mechanobiology of malignant brain tumors using a brain matrix-mimetic hyaluronic acid hydrogel platform. Biomaterials, 32, 7913-7923.

116. Fedrigo, C. A., Grivicich, I., Schunemann, D. P., et al. (2011). Radioresistance of human glioma spheroids and expression of HSP70, p53 and EGFr. Radiation Oncology, 6, 156.

117. Jin, S. G., Jeong, Y. I., Jung, S., Ryu, H. H., Jin, Y. H., \& Kim, I. Y. (2009). The effect of hyaluronic acid on the invasiveness of malignant glioma cells : comparison of invasion potential at hyaluronic acid hydrogel and matrigel. Journal of Korean Neurosurgical Society, 46, 472-478.

118. Coquerel, B., Poyer, F., Torossian, F., et al. (2009). Elastin-derived peptides: matrikines critical for glioblastoma cell aggressiveness in a 3-D system. Glia, 57, 1716-1726. 